\title{
NELSON MACULAN FILHO: CIENTISTA E EDUCADOR
}

\section{Celso Carneiro Ribeiro}

Pontifícia Univ. Católica do Rio de Janeiro (PUC-Rio)

Rio de Janeiro - RJ

\section{Mário Veiga Ferraz Pereira}

Power Systems Research (PSR)

Rio de Janeiro - RJ

\section{Nair Maria Maia de Abreu *}

Coordenação dos Programas de Pós-Graduação de Engenharia (COPPE/UFRJ)

Universidade Federal do Rio de Janeiro

Rio de Janeiro - RJ

nair@pep.ufrj.br

\author{
Ruy Eduardo Campello \\ FURNAS Centrais Elétricas S.A. e \\ Instituto Metodista Bennett (IMB) \\ Rio de Janeiro - RJ \\ * Corresponding author/autor para quem as correspondências devem ser encaminhadas
}

Recebido em 06/2002, aceito em 11/2002

\begin{abstract}
Resumo
Este texto é um pequeno e merecido tributo ao Professor Nelson Maculan Filho por ocasião do seu $60^{\circ}$ aniversário, em reconhecimento às suas grandes contribuições à Universidade brasileira. Como amigos, colaboradores e ex-alunos, ficamos sensibilizados e honrados com o convite feito pelo Professor Jayme Luiz Szwarcfiter para escrever este artigo. Carpe diem, querido amigo Maculan!
\end{abstract}

Palavras-chave: Nelson Maculan; otimização combinatória; programação inteira; matemática aplicada.

\begin{abstract}
This article is a small and well deserved tribute to Professor Nelson Maculan Filho on occasion of his $60^{\text {th }}$ anniversary, in recognition to his outstanding contributions to Brazilian universities. As friends, collaborators and former students, we are truly heartier and honored to be invited by Professor Jayme Luiz Szwarcfiter to write down this work. Carpe diem, dear friend Maculan!
\end{abstract}

Keywords: Nelson Maculan; combinatorial optimization; integer programming; applied mathematics. 


\section{Introdução}

A tentativa de resumir em um artigo a atuação do amigo e colega Nelson Maculan Filho como pesquisador e, principalmente, como educador, notável por sua amplitude e impacto, é uma tarefa árdua. Diante de tamanha responsabilidade e indisfarçável emoção, é natural a intimidação e a preocupação com trocas de datas e omissões de fatos, publicações e detalhes importantes, ainda mais que, lamentavelmente, a memória torna-se caprichosa e traiçoeira com o tempo. Nossa disposição é a de reunir fatos e lembranças que possam ilustrar a sua notável atuação para aqueles que não tiveram o privilégio de seu convívio. Ou então é, simplesmente, uma forma de recordação carinhosa para os afortunados como nós que tivemos e continuamos tendo esta oportunidade.

Nelson Maculan Filho nasceu em Londrina, Paraná, no dia 19 de março de 1943. Formou-se Engenheiro de Minas e Metalurgia pela Escola de Minas de Ouro Preto em Minas Gerais em 1965. Um ano depois voou para a França, para iniciar sua carreira científica. Em 1967, sob a orientação do Professor Jean Abadie, concluiu seu DEA em Estatística-Matemática na Université Paris VI, com um memorial sobre programação não-linear inteira. Nas barricadas de maio de 1968 conheceu Anne Marie Delaunay, francesa do Jura, com quem se casou. Pouco tempo depois, retornou ao Brasil. Assim, Nelson Maculan escolheu seu país natal para também se tornar um cientista brasileiro. Foi uma escolha corajosa, para quem teve oportunidade de ser cientista com certidão de primeiro mundo.

Obteve seu doutorado na COPPE/UFRJ em 1975, com a tese "Modelo matemático para expansão de uma rede de telecomunicações". Com outra tese, intitulada "Problema de Steiner em grafos" e defendida em 1988 para a obtenção de sua Livre Docência na UFRJ, tratou e divulgou entre nós este importante problema, até então pouco conhecido entre nossos pesquisadores e alunos da área de Otimização Combinatória. E, assim, seguiu o Maculan levando na bagagem, no colo, e até mesmo no bolso, alunos e colegas, abrindo para muitos deles a porta do saber científico. Desde seu retorno ao Brasil, foram mais de 100 orientações de dissertações de mestrado e quase 40 teses de doutorado, entre brasileiros e latino-americanos. Muitos destes, por sua vez, orientaram novos alunos, formaram novas gerações de cientistas (como podemos observar na sua árvore de orientados no Anexo III) e seguem distribuindo a sabedoria do mestre e colaborando na construção e no desenvolvimento da América Latina. Suas atividades de ensino e pesquisa foram reforçadas pela sua sempre presente atuação em sociedades científicas como SOBRAPO, SBC, SBMAC e ALIO, citando-se ainda sua constante participação em comitês científicos e agências de fomento, tais como CNPq, CAPES, FINEP e FAPERJ.

Sua produção científica ultrapassa hoje uma centena de trabalhos publicados em periódicos, congressos e coletâneas, dos quais os principais são destacados no Anexo I. Seus trabalhos percorrem a Programação Linear, desde o método do Simplex até o algoritmo de Karmarkar. Suas principais contribuições estão associadas a métodos de corte e a técnicas de decomposição e de relaxação lagrangeanas, assim como ao problema de Steiner em grafos e suas variantes. Outras contribuições em programação inteira e otimização combinatória aparecem em trabalhos sobre problemas como os da mochila, de corte, de particionamento, de escalonamento e de otimização em grafos. Sua produção registra ainda aplicações em áreas como energia, finanças, psiquiatria, ecologia, transporte e distribuição, petróleo, agro-indústria e pesca, cartografia e telefonia. Como se isto não bastasse, Nelson Maculan é autor e editor de diversos livros e também autor de muitos capítulos de livros publicados por importantes editoras internacionais. 
Na próxima seção, serão descritas as principais atividades e realizações de Nelson Maculan Filho na UFRJ, destacando-se sua atuação como Reitor. A Seção 3 aborda sua trajetória e algumas de suas principais contribuições na área de Otimização Combinatória, na qual se concentrou a maior parte de suas atividades de pesquisa. Como não dispomos de espaço para abordar suas contribuições, limitamo-nos a apresentar no Anexo I uma lista de suas principais publicações. Na Seção 4 comentamos sua atuação na sociedade e na comunidade científica, em especial no Brasil e na América Latina. Comentários finais sobre sua visão humanista e sua natureza aberta e democrática são apresentados na Seção 5, que é seguida por três anexos incluindo suas principais publicações, depoimentos de muitos de seus ex-orientados e a "árvore" de seus orientados de doutorado.

\section{Maculan e a UFRJ}

Nelson Maculan ingressou na UFRJ como Professor Assistente da COPPE e do Instituto de Matemática em 1971, passando a Adjunto em 1978. Em 1989, tornou-se Professor Titular do Programa de Engenharia de Sistemas e Computação da COPPE. Durante todos estes anos e até os dias de hoje ele atuou na graduação e na pós-graduação do Instituto de Matemática, onde leciona diversas disciplinas, entre as quais Cálculo, Cálculo Numérico, Probabilidade e Estatística, Álgebra Linear, Computação e Pesquisa Operacional. No entanto, notável é sua atuação no Programa de Engenharia e Sistemas e Computação da COPPE. Lá, Maculan respira e leva oxigênio para muitos de seus alunos e colegas. Funciona como um verdadeiro motor, não só lecionando diferentes disciplinas, como Programação Linear e Inteira, Otimização em Grafos, Otimização de Grande Porte, Otimização Combinatória, Álgebra Linear Computacional e Simulação, mas, sobretudo, liderando projetos e grupos de pesquisa e exercendo diferentes cargos de administração acadêmica. Sua vida acadêmica está longe de se limitar à condição de excelente professor e pesquisador. Com apenas dois anos de universidade, assumiu a coordenação do Programa de Engenharia de Sistemas e Computação. A partir daí, passou por diversas funções até ser eleito e tornar-se Diretor da COPPE em 1990. Exerceu esta função por um curto período, sendo no mesmo ano levado pelo voto dos professores, alunos e funcionários ao cargo máximo da UFRJ. O Magnífico Reitor Nelson Maculan Filho passou então a administrar a maior universidade federal brasileira.

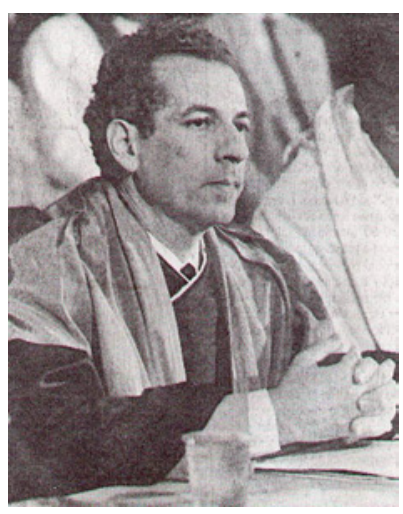

Cercaram-lhe então mais de 23.000 alunos e 13.000 docentes e funcionários técnico-administrativos, além de toda a infraestrutura física do campus da UFRJ no Rio de Janeiro. Nelson Maculan chegava para devolver à UFRJ o equilíbrio em torno do ensino e da pesquisa, distribuir melhor a relação entre funcionários e alunos e liderar o aumento da produção científica, sem perda de sua essência. Os resultados de sua administração foram expressivos - informações obtidas do "Relatório de Gestão da Reitoria da UFRJ, 1990-1994" (coordenação editorial de Deolinda Cavalcante Saraiva, Frilas Serviços Jornalísticos, Imprinta Gráfica e Editora). Em 1994, deixou a Reitoria com uma Universidade constituída por 3.400 docentes, 10.500 funcionários e 35.100 alunos, dos quais 25.000 eram de graduação, 9.000 de pós-graduação e o restante 1.100 constituídos por estudantes do Colégio de Aplicação e de cursos técnicos da Escola de Música. Nesta ocasião, a área de ensino oferecia 55 cursos de graduação e 135 de pós-graduação stricto sensu (mestrado e doutorado), além de dezenas de cursos de especialização, das turmas de primeiro 
e segundo graus e dos cursos técnicos da Escola de Música. Nos cursos de doutorado foi constatado um crescimento de $27 \%$ do número de alunos. Na área de pesquisa, além das teses em preparação, Nelson Maculan deixou a UFRJ com mais de 2.000 projetos em andamento. Destes projetos, muitos vinham de intensa cooperação internacional, a exemplo do convênio com o Centro Europeu de Física de Altas Energias (CERN) que ainda vem trazendo excelentes resultados em novas pesquisas em Física, Informática e Engenharia Elétrica. Diversas pesquisas importantes ficaram em andamento nas áreas de Saúde (visando, por exemplo, a prevenção de doenças infecto-contagiosas), Robótica e Engenharia Civil (que resultaria em desenvolvimento de tecnologia inédita, genuinamente brasileira, para exploração de petróleo em águas profundas) e ainda em Ecologia (no estudo da poluição ambiental provocada pelo mercúrio no Rio Tapajós).

Cabe registrar que, mesmo enquanto exercia a função máxima da universidade, Maculan fez questão de não abandonar a sala de aula. Também não deixou de atender seus orientandos, como podemos constatar pelos depoimentos transcritos no Anexo II, e ainda encontrou tempo para concluir um livro com um de seus colaboradores.

Com elevado sentido de responsabilidade social, o Reitor se preocupou com a garantia do ensino público, em áreas socialmente estratégicas, acessível aos segmentos da sociedade que dependem da opção noturna para estudar. Assim, na sua gestão a UFRJ se preparou para oferecer o ingresso a 6.051 alunos dos cursos noturnos, distribuídos entre aqueles de Química, Matemática, Física, Serviço Social, Fonoaudiologia, Fisioterapia, Biologia, Dança, Educação Física, Direção Teatral, Radialismo e Televisão.

$\mathrm{Na}$ próxima seção ilustraremos sua atuação como cientista, abordando sua trajetória e algumas de suas principais contribuições para a área de Otimização Combinatória, na qual se concentrou a maior parte de suas atividades de pesquisa e onde ainda vem atuando de forma sempre presente.

\section{Iniciativas em Otimização Combinatória}

No início da década de 1970, a Coordenação dos Programas de Pós-Graduação em Engenharia - COPPE/UFRJ destacava-se, como até hoje, como instituição de enorme responsabilidade e prestígio no âmbito científico. Recém-chegado da França e contratado, o jovem Professor Assistente Nelson Maculan, então com 28 anos, encontrou na instituição terreno fértil para desenvolver sua curiosidade e criatividade como pesquisador. Destacou-se de pronto pela maneira cativante e informal com que, sem abdicar do rigor científico, ministrava suas aulas. Também chamava atenção pela cortesia com que se relacionava com seus alunos, em uma época em que os protocolos acadêmicos ainda seguiam padrões mais convencionais. Por mais ocupado que estivesse, Maculan nunca se esquecia dos pedidos de seus alunos, nem se furtava a atendê-los em sua casa em "momentos de crise" ou de falta de tempo.

Sempre atento aos últimos desenvolvimentos, de pronto mergulhou nas novas teorias e tendências científicas de que se tinha notícia. Criou um ativo programa de intercâmbio com pesquisadores internacionais. Entre os principais pesquisadores que convidou e acolheu na COPPE, podemos citar Egon Balas, Ellis Johnson, Eugene Lawler e Michel Minoux. Os cursos que ministraram foram marcos de grande importância para o desenvolvimento da área de Otimização Combinatória no Brasil e para a abertura de espírito de seus alunos e colegas. 


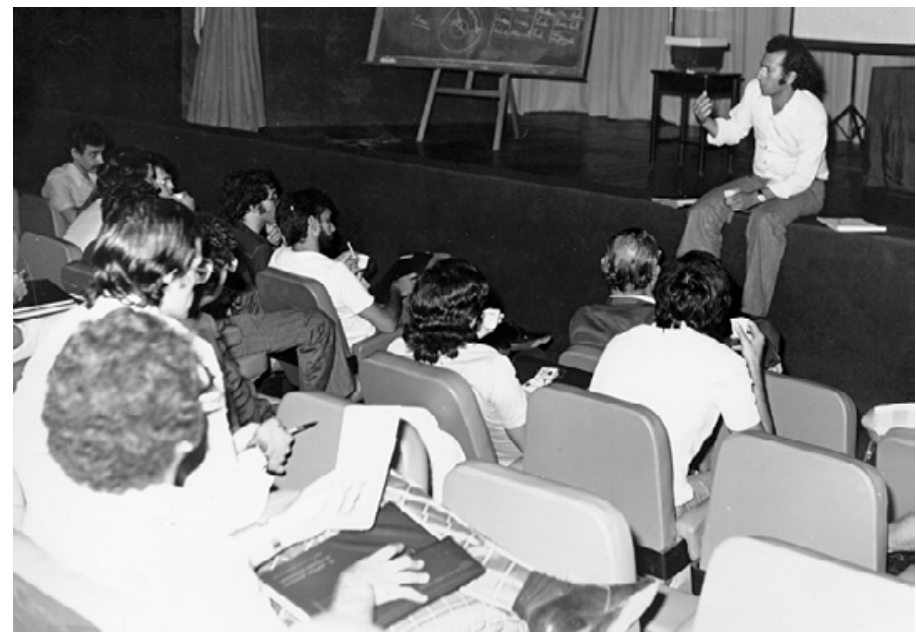

Nelson Maculan em apresentação no VIII Simpósio Brasileiro de Pesquisa Operacional, realizado em Recife de 17 a 20 de setembro de 1975.

Numa de suas primeiras linhas de atuação, Maculan compartilhou a percepção, simples em essência, de que problemas de programação matemática não convexa, quando estruturados na forma linear com condições lógicas, expõem informações sobre a estrutura particular de cada problema que podem então ser utilizadas na busca por soluções ótimas. Diversos problemas de relevância prática como o da complementaridade linear, o da programação quadrática, os de programação de eventos e os de particionamento e de recobrimento, entre outros, quando colocados no formato disjuntivo dão origem a surpreendentes possibilidades para o desenvolvimento de algoritmos. Os fundamentos teóricos da Programação Disjuntiva, bem como os resultados mais importantes, são fruto das pesquisas dos professores Egon Balas (Carnegie-Mellon University) e Robert G. Jeroslow ${ }^{\dagger}$ (1942-1988), dois gigantes da área de Otimização Combinatória.

Percebendo a importância e a oportunidade deste novo tema para a área de Otimização Combinatória, pesquisador curioso, atento e sempre buscando conhecimentos de vanguarda, convidou o professor Balas para ministrar um curso de Programação Disjuntiva no Programa de Engenharia de Sistemas e Computação da COPPE/UFRJ em 1978. Os benefícios desta iniciativa foram significativos e puderam ser mensurados nos anos seguintes pelas teses e publicações de brasileiros no tema. Com sua visão científica ampla, Nelson Maculan não se limitou a criar algoritmos e produzir apenas resultados teóricos. Com o apoio de seus colaboradores, explorou e implementou algoritmos combinando cortes disjuntivos e enumeração implícita para problemas de particionamento, de recobrimento e de complementaridade linear, orientando dissertações de mestrado e teses de doutorado. Confirmando a visão prospectiva e isenta de preconceito do pesquisador e educador, é interessante recordar que estes algoritmos mistos eram pouco explorados naquela época, em função da grande resistência do meio científico ao reconhecimento de seu mérito computacional, sendo considerados como pouco elegantes. Como resultado deste ceticismo, dificilmente alcançavam espaço nas publicações científicas indexadas. Alguns anos mais tarde, porém, provaram de forma definitiva sua utilidade, resultando eficientes na solução de grandes instâncias de problemas de otimização combinatória. 
Nelson Maculan atuou como Professor Visitante da Université de Montréal (Canadá) durante seu primeiro ano sabático (1982-1983). Na mesma ocasião, Ruy Campello, um de seus muitos colaboradores e ex-alunos, estava em período de pós-doutoramento na CarnegieMellon University (Estados Unidos). Costumavam manter conversas freqüentes por telefone. Durante o ríspido inverno, discutiam sobre a oportunidade de produção de um livro, em Português, abordando algoritmos heurísticos e sua garantia de performance para problemas NP-Árduos, assim como esquemas de aproximação polinomiais e completamente polinomiais. Acreditavam que pelo conteúdo e especialmente por ser em Português, poderia resultar em contribuição útil para apoiar cursos de graduação e de pós-graduação no Brasil. Dedicaramse então com afinco a esta tarefa, que só estaria concluída após o retorno de ambos ao Brasil, e não antes de contornarem inúmeras dificuldades, como a falta de recursos e apoio institucional para garantir uma publicação em condições dignas. Neste episódio permanece iluminado na memória dos autores a fé, a grande generosidade e o apoio recebidos da saudosa professora Doris Ferraz de Aragon $^{\dagger}$ (1936-2000), que não poupou esforços para viabilizar a publicação. Após muita ajuda dos amigos, com perseverança e um grande atraso, enfim foi publicado em 1994 o livro "Algoritmos e Heurísticas: Desenvolvimento e Avaliação de Performance”. Passados quase dez anos, este texto ainda é utilizado em cursos de pós-graduação e graduação em Universidades de primeira linha no Brasil.

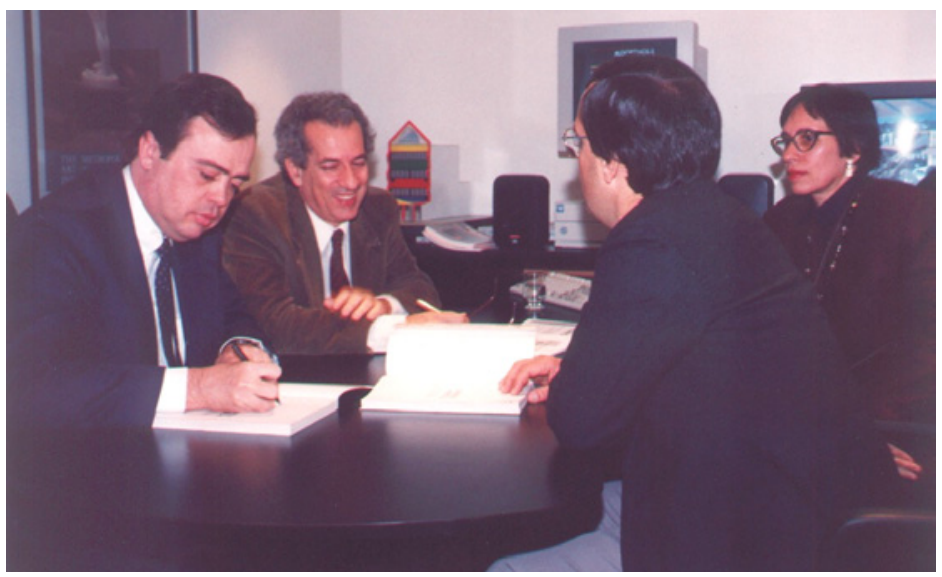

Por ocasião do lançamento do livro "Algoritmos e Heurísticas:

Desenvolvimento e Avaliação de Performance": professores Ruy Campello, Nelson Maculan, Edmundo Souza e Silva e Nair Abreu (1994).

Outra importante contribuição científica de Nelson Maculan diz respeito à sua formulação para o problema de Steiner em grafos, descrita a seguir. Dados um grafo não-orientado $\mathrm{G}=(\mathrm{V}, \mathrm{E})$, onde $\mathrm{V}$ é o conjunto de vértices e $\mathrm{E}$ é o conjunto de arestas, custos não-negativos associados às suas arestas em um subconjunto $\mathrm{T} \subseteq \mathrm{V}$ de nós terminais, define-se uma árvore de Steiner como uma sub-árvore G que contenha todos os vértices terminais. Seu custo é dado pela soma dos custos de suas arestas. O problema de Steiner em grafos consiste em determinar-se uma árvore de Steiner de custo mínimo. Em trabalho conjunto realizado com o pesquisador Armin Claus, Maculan propôs uma reformulação deste problema através de um digrafo obtido a partir do grafo G. Esta formulação foi baseada em uma formulação também 
introduzida por ele para o problema da árvore geradora direcionada enraizada no nó s, que usa um número polinomial de variáveis e de restrições e cuja relaxação linear fornece a solução ótima inteira para o problema. A idéia central da formulação proposta para o problema de Steiner em grafos consiste em enviar a partir de um nó s, escolhido como raiz, uma unidade de fluxo de uma mercadoria distinta para cada vértice terminal em $\mathrm{T}-\{\mathrm{s}\}$. Esta reformulação proposta por Armin Clauss e Nelson Maculan também foi introduzida independentemente por John Beasley e por Richard Wong. Ela vem sendo amplamente utilizada na literatura para resolver o problema de Steiner em grafos e algumas generalizações. $\mathrm{O}$ mesmo se aplica à formulação proposta por Nelson Maculan para o problema da árvore geradora s-direcionada. Deve-se mencionar que seu trabalho foi desenvolvido numa época em que o problema de Steiner ainda era pouco conhecido e explorado. Hoje, este problema é amplamente estudado devido às suas importantes aplicações em problemas de projeto de redes de comunicação e tem recebido contribuições importantes de diversos pesquisadores brasileiros, que propuseram alguns dos melhores algoritmos exatos e heurísticos atualmente disponíveis para sua solução.

Retrocedendo no tempo nessa narrativa, há outra contribuição importante sua para o desenvolvimento da área de Otimização Combinatória no Brasil que não poderia ficar esquecida: a organização da School on Combinatorial Optimization (Rio de Janeiro, 8 a 19 de julho de 1985). Este evento internacional reuniu pesquisadores de inegável talento, brasileiros e do Exterior, de países como Inglaterra, França, Itália, Polônia, Áustria, Holanda, Bélgica, Espanha, Portugal, Suíça, Suécia, Estados Unidos, Canadá, México, Equador, Argentina, Chile, Uruguai, Peru, Venezuela e Colômbia.

Era necessário impulsionar a área de Otimização Combinatória no Brasil e nada mais oportuno do que um encontro desta qualidade e magnitude. A escolha do local de realização foi extremamente significativa. O mais simples seria um belo hotel na orla marítima do Rio, em função das dificuldades logísticas que um evento desta natureza traria, especialmente pelo comparecimento de bom número de pesquisadores estrangeiros que em sua maioria jamais haviam estado no Brasil. Nelson Maculan ousou mais uma vez. O evento foi realizado na UFRJ, pois, desta forma, a contribuição seria muito mais relevante e um autêntico marco acadêmico, dando amplo acesso à participação de estudantes de diversos programas de pósgraduação. Alguns professores brasileiros também foram convidados para apresentar cursos e trabalhos, dando-lhes a oportunidade de divulgar suas contribuições no Exterior. Não poderia estar mais certo: os resultados alcançados pelo evento foram amplamente compensadores. Decorridos tantos anos da realização da School on Combinatorial Optimization, esta Escola ainda é reconhecida como um marco do desenvolvimento da área no Brasil. Como resultado deste evento e dos cursos nele ministrados, foi publicado o livro "Surveys in Combinatorial Optimization" editado por Silvano Martello, Gilbert Laporte, Michel Minoux e Celso Ribeiro (North-Holland, 1987), que ainda permanece como uma referência importante na área. 


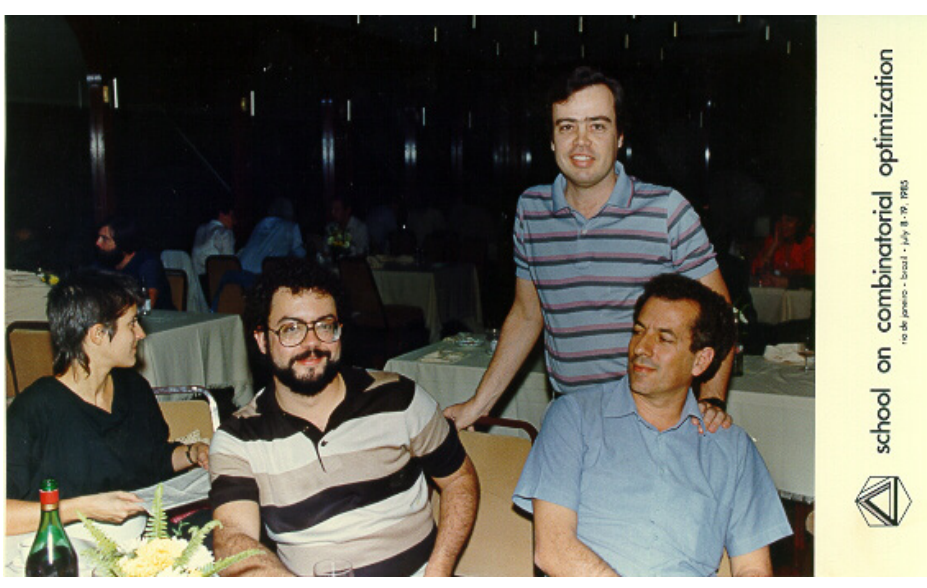

Por ocasião do jantar de confraternização no Pão de Açúcar, os organizadores da

School on Combinatorial Optimization: professores Celso Carneiro Ribeiro, Nelson Maculan (sentados) e Ruy Campello (1985).

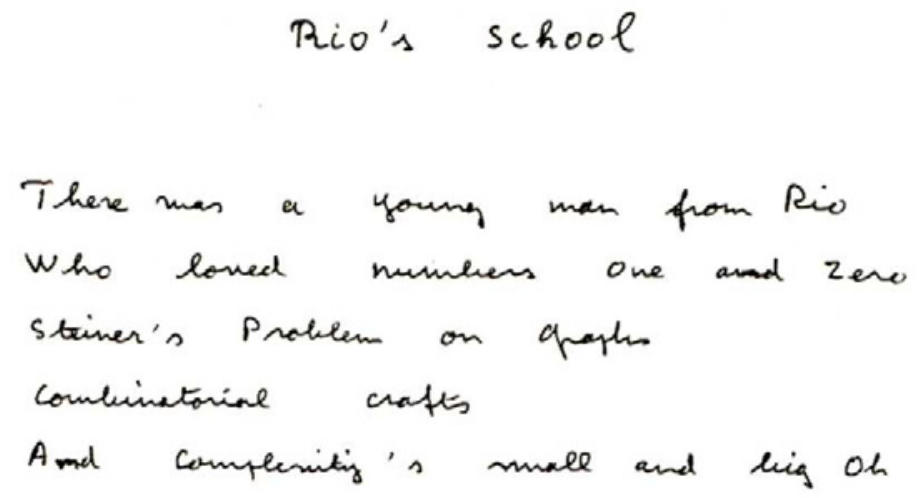$$
\text { Rio's school }
$$$$
\text { There mas a young man from Rio }
$$$$
\text { who loved numbers one and zero }
$$$$
\text { stainer's Problem on qraph }
$$$$
\text { combinatoriace crafts }
$$$$
\text { And complesitin's mable and lig oh }
$$

Agradecimento de Pierre Hansen em nome dos participantes da School on Combinatorial Optimization (1985). 


\section{Atuação na sociedade e na comunidade científica}

Desde seu retorno ao Brasil em 1971, Nelson Maculan teve e continua tendo uma atuação marcante na sociedade e na comunidade científica nacional e latino-americana. Participou e envolveu-se com diversas sociedades científicas brasileiras, sendo por exemplo sócio fundador da Sociedade Brasileira de Computação (SBC). A título ilustrativo, resumimos sua trajetória na área de Pesquisa Operacional.

Após ter sido um dos primeiros sócios da Sociedade Brasileira de Pesquisa Operacional (SOBRAPO), foi escolhido como seu Diretor-Presidente para o período 1975-1976. A preocupação inicial de sua diretoria foi a transferência definitiva para o Rio de Janeiro da sede da SOBRAPO e o registro de seus estatutos (Zilma E. Lóss, O Desenvolvimento da Pesquisa Operacional no Brasil, Dissertação de Mestrado, Programa de Engenharia de Produção, COPPE/UFRJ, 1981). A idéia de realizar um congresso latino-americano de Pesquisa Operacional surgiu em 1981, em conversas com Andres Weintraub (Chile) e Hugo Scolnik (Argentina) durante um encontro no Rio de Janeiro. Assim sendo, foi realizado e organizado no Rio de Janeiro em outubro de 1982 o I Congresso Latino-Ibero-Americano de Pesquisa Operacional e Engenharia de Sistemas (I CLAIO), em conjunto com o XV Simpósio Brasileiro de Pesquisa Operacional. Apenas 12 dos assistentes compareceram com o objetivo específico de participar do I CLAIO, contrastando com a participação de centenas de assistentes e conferencistas nas realizações bienais recentes do evento, que ilustram sua pujança e a clarividência de seus criadores e dos continuadores desta iniciativa. Este I CLAIO também marcou a fundação da ALIO - Asociación Latino-Ibero-Americana de Investigación Operativa, que teve o professor Roberto Galvão (Universidade Federal do Rio de Janeiro) como seu primeiro presidente. Nelson Maculan viria posteriormente a presidir a ALIO em duas ocasiões, com mandatos bienais nos períodos 1988-1990 e 1998-2000. Viria também a ser Vice-Presidente da IFORS - International Federation of Operational Research Societies de 1983 a 1985. Também criou e foi o primeiro Editor-Chefe da revista Investigación Operativa publicada pela ALIO, que fundiu-se recentemente ao periódico International Transactions in Operations Research publicado pela IFORS e que tem Celso Ribeiro como seu Editor Regional para a América Latina.

O cientista e educador Nelson Maculan acumulou prêmios e distinções ao longo de sua carreira: Medalha da Ordem Nacional do Mérito Educativo (no grau de Comendador, 1994), Chevalier dans l'Ordre des Palmes Académiques (França, 1998), IFORS Distinguished Lecturer (2000) e, mais recentemente, a Medalha da Ordem Nacional do Mérito Científico (no grau de Comendador, 2002). É Membro Titular da Academia Brasileira de Ciências, da Academia Nacional de Engenharia e da Academia Européia de Ciências, Letras e Humanidades.

\section{A pessoa e o humanista}

Além de cientista e educador, Nelson Maculan tem uma vasta cultura e é um ávido leitor nas mais diversas áreas do conhecimento. Tem uma visão humanista da Sociedade e uma natureza democrática e sempre aberta ao diálogo, à colaboração, ao entendimento e à conciliação.

Profundamente engajado na defesa dos direitos do Homem e nas ações pela cidadania, teve uma atuação destacada na época mais negra da repressão política. Como responsável acadêmico, esteve sempre ao lado de alunos e colegas da UFRJ na defesa de seus direitos individuais e na preservação de suas liberdades. Não foram poucas as ocasiões em que 
interveio pessoalmente pela libertação de alunos presos ou perseguidos. Muitas foram as vezes em que foi despertado com a notícia de uma prisão ou do desaparecimento de alunos, colegas e amigos. Muitos foram aqueles que ajudou a resgatar.

De forma recorrente é lembrada a importância da família como esteio, fonte de inspiração, emoção e motivação na vida dos indivíduos. Por ocasião do sexagésimo aniversário do amigo Maculan, celebração muito especial, não poderíamos deixar de estender nosso carinho à Anne-Marie e seus três filhos, que também sempre nos receberam com carinho em sua casa e junto aos quais também compartilhamos muitos momentos importantes.

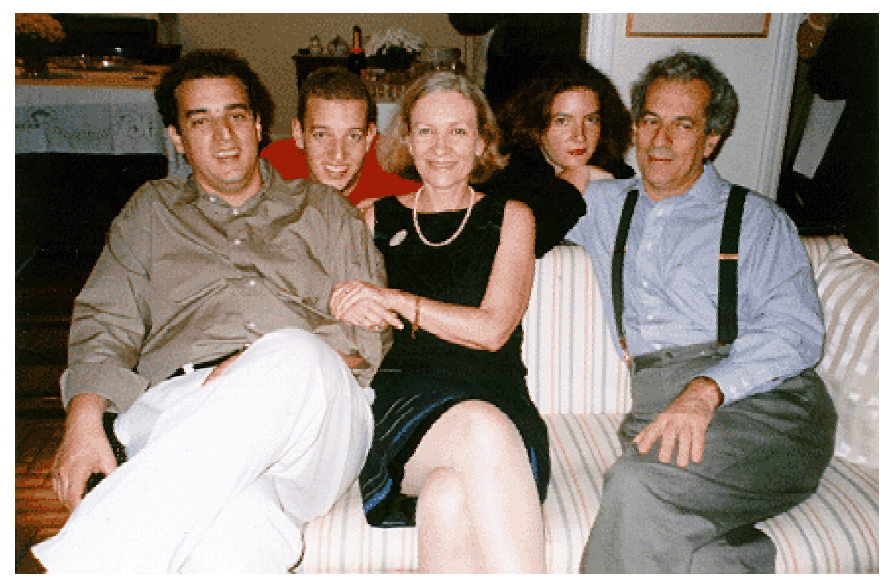

Os Maculan: Julien, Sebastien, Anne-Marie, Berenice e Nelson. 


\section{Anexo I. Principais publicações}

(1) Alencar, T.G.; Maculan, N. \& Oliveira, A.A. (1978). Uma caracterização algébrica do conjunto de definições do problema de programação inteira. Anais do XI Simpósio Brasileiro de Pesquisa Operacional.

(2) Albuquerque, R. \& Maculan, N. (1979). Alguns tópicos sobre a programação linear estocástica. Anais do XII Simpósio Brasileiro de Pesquisa Operacional.

(3) Aloise, D.J. \& Maculan, N. (1993). Uma classe de algoritmos aproximativos decrescentes para o problema "bin-packing". Revista Brasileira de Computação, 6, 3-12.

(4) Alvarenga, A.G. \& Maculan, N. (1986). Um método aproximado para a solução do problema de cortes de placas a duas dimensões. Revista Brasileira de Engenharia, Caderno de Engenharia Naval, 3, 79-90.

(5) Antonelli, P.; Maculan, N.; Portugal, R.; Rutz, S. \& Sabau, S. (2001). Transient-states analysis of a 2-species dynamical ecological model via KCC-theory. Nonlinear Studies, 8, 283-296.

(6) Barbosa, H.; Raupp, F.; Lavor, C.; Lima, H. \& Maculan, N. (2000). A hybrid genetic algorithm for finding stable conformations of small molecules. Proceedings of the $V^{\text {th }}$ Brazilian Symposium on Neural Networks, 1, 90-94.

(7) Borges, P.R.T.; Pirin, C. \& Maculan, N. (1980). Contribuição aos problemas de programação linear com variáveis limitadas de maneira explícita. Anais do III Congresso Brasileiro de Automática, 159-164.

(8) Braga, L.P.V. \& Maculan, N. (1981). Aspectos computacionais em programação linear. Revista Brasileira de Computação, 1, 19-39.

(9) Braga, L.P.V. \& Maculan, N. (1983). Kriging theory and applications for automated cryptography. Anais do XVI Simpósio Brasileiro de Pesquisa Operacional, 698-708.

(10) Braga, L.P.V. \& Maculan, N. (1984). Métodos computacionais e estatísticos em cartografia geológica. Anais do VI Simpósio Brasileiro de Probabilidade e Estatística, 241-248.

(11) Campello, R.E. \& Maculan, N. (1976). Branch \& bound approach to a fixed-charge network expansion. Memórias Matemáticas, 82.

(12) Campello, R.E. \& Maculan, N. (1984). On deep disjunctive cutting planes for set partitioning: A computationally oriented research. In: Mathematical Programming [edited by R. Cottle, M. Kelmanson and B. Korte], 69-78, North Holland.

(13) Campello, R.E. \& Maculan, N. (1987). Lagrangean relaxation for a lower bound to a set partitioning problem with side constraints. Discrete Applied Mathematics, 18, 119-136.

(14) Campello, R.E. \& Maculan, N. (1988). An O(n $\left.{ }^{3}\right)$ worst case bounded special LP knapsack (0-1) with two Constraints. RAIRO Recherche Opérationnelle, 22, 27-32.

(15) Campello, R.E. \& Maculan, N. (1994). Algoritmos e heurísticas: Desenvolvimento e avaliação de performance. EDUFF, Niterói. 
(16) Candia Vejar, A. \& Maculan, N. (1992). Algoritmos de reducción para el problema de Steiner en dígrafos. Trabajos Seleccionados del V CLAIO - Congreso Latino-IberoAmericano de Investigación Operativa e Ingeniería de Sistemas, 63-70.

(17) Caprihan, A. \& Maculan, N. (1976). Sampling from distributions using piecewise functional approximation. Anais do II Simpósio Brasileiro de Probabilidade $e$ Estatística, 50-62.

(18) Cazalis, D. \& Maculan, N. (1989). Projective purification algorithm. Anais do XII Congresso Nacional de Matemática Aplicada e Computacional, 156-160.

(19) Charifker, I.; Rosenthal-Matos, R. \& Maculan, N. (1975). Um problema de programação linear de grande porte: Expansão de redes de telecomunicação. Anais do VIII Simpósio Brasileiro de Pesquisa Operacional.

(20) Fampa, M. \& Maculan, N. (2001). A new relaxation in conic form for the euclidean Steiner problem in $\mathrm{R}^{\mathrm{n}}$. RAIRO Recherche Opérationnelle, 35, 383-394.

(21) Figueiredo, J.N.; Gonzaga, C.C. \& Maculan, N. (1979). Localização ótima de torres de transmissão utilizando teoria dos grafos. Pesquisa Operacional, 2, 12-22.

(22) Figueiredo, R. \& Maculan, N. (2000). O problema do caixeiro viajante assimétrico: Limites inferiores e um novo algoritmo de separação. Investigação Operacional, 20, 127-146.

(23) Fortunato, L.A.M.; Campello, R.E. \& Maculan, N. (1987). Coordenação dos cronogramas de manutenção preventiva de unidades geradoras do sistema interligado brasileiro. Anais do IX Seminário Nacional de Produção e Transmissão de Energia Elétrica, BH/GOP/03.

(24) Galvão, R.D.; Jacobsen, S.K.; Maculan, N. \& Maffioli, F. (editors, 1992). Special issue on Practical Combinatorial Optimization. European Journal of Operational Research, 58.

(25) Gomes, F.J.; Thomaz, A.C.F. \& Maculan, N. (1984). Distribuição ótima da idade como determinante na pesca de atuns. Anais do XVIII Simpósio Brasileiro de Pesquisa Operacional, 362-371.

(26) Hoto, R.; Maculan, N.; Marques, F. \& Arenales, M. (2003). Um problema de corte com padrões compartimentados. Pesquisa Operacional, 23(1), 169-187.

(27) Jardim Campos, M.H. \& Maculan, N. (1995). Optimization problems related to the cut of paper reels: A dual approach. Investigación Operativa, 5, 45-53.

(28) Jardim Campos, M.H. \& Maculan, N. (1988). Problemas de otimização relacionados ao corte de bobinas de papel. Anais do XVI Colóquio Brasileiro de Matemática, 159-182.

(29) Lavor, C. \& Maculan, N. (2001). Interval analysis applied to global minimization of potential energy function. In: Advances in Convex Analysis and Global Optimization [edited by N. Hadljisavvas and P. Pardalos], Noncovex Optimization and its Applications, 54, 333-344, Kluwer.

(30) Lavor, C.C. \& Maculan, N. (2002). A function to test methods applied to global minimization of potential energy of molecules. Numerical Algorithms, a ser publicado.

(31) Lomba, L.F. \& Maculan, N. (1988). Algoritmo O(n) para o problema de programação linear da mochila (knapsack) 0-1. Pesquisa Operacional, 8, 1-19. 
(32) Maculan, N. (1981). Algoritmo de Khachiyan para a resolução de problemas de programação linear. Noticiário da Sociedade Brasileira de Matemática, 2, 28-37.

(33) Maculan, N. (1983). Relaxation Lagrangienne: le problème du knapsack 0-1. INFOR, 21, 315-327.

(34) Maculan, N. (1986). A new linear programming formulation for the shortest s-directed spanning tree problem. Journal of Combinatorics, Information and System Sciences, 11, 53-56.

(35) Maculan, N. (1986). A linear programming for the shortest s-directed tree problem. Actes des Journées du 20ème Anniversaire du Groupe Combinatoire de l'AFCET, 287-396.

(36) Maculan, N. (1986). O problema de Steiner em grafos. Avances en Investigación Operativa, 206-213, SADIO, Buenos Aires.

(37) Maculan, N. (1987). The Steiner problem in graphs. In: Surveys in Combinatorial Optimization [edited by S. Martello, G. Laporte, M. Minoux and C.C. Ribeiro], Annals of Discrete Mathematics, 31, 185-212.

(38) Maculan, N. (1988). Linear programming problem for which Karmarkar's algorithm is strongly polynomial. Pesquisa Operacional, 8, 52-55.

(39) Maculan, N. (1990). Combinatorial Optimization in Computer Networks. In: Science Policy Research - Implications and Applications [edited by D. de Almeida], 69-75, Pinter Publishers.

(40) Maculan, N. (2002). Linear 0-1 programming. In: Handbook of Applied Optimization [edited by P.M. Pardalos and M.G.C. Resende], 440-445, Oxford University Press.

(41) Maculan, N. (2002). Introduction to integer programming. In: Handbook of Applied Optimization [edited by P.M. Pardalos and M.G.C. Resende], 431-439, Oxford University Press.

(42) Maculan, N. \& Paula Jr., G.G. de (1989). A linear-time median-finding for projecting a vector on the simplex of $\mathrm{R}^{\mathrm{n}}$. Operations Research Letters, 8, 219-222.

(43) Maculan, N.; Arpin, D. \& Nguyen, S. (1988). Le problème de Steiner sur un graphe orienté: Formulations et relaxations. Computational and Applied Mathematics, 7, 109-118.

(44) Maculan, N.; Michelon, P. \& Xavier, A.E. (2000). The Euclidian Steiner tree problem in $\mathrm{R}^{\mathrm{n}}$ : A mathematical programming formulation. Annals of Operation Research, 96, 209-220.

(45) Maculan, N.; Minoux, M. \& Plateau, G. (1997). A $O(n)$ algorithm for projecting a vector on the intersection of a hyperplane and $\mathrm{R}^{\mathrm{n}}+$. RAIRO Recherche Opérationnelle, 31, 7-16.

(46) Maculan, N. \& Netto, M.A.C. (2001). Alianças para o desenvolvimento do pensamento logístico e gerenciamento da multimodalidade. In: Transportes Experiências em Rede [edited by C. Nassi, A. Brasileiro, E. Kawamoto and L.A. Lindau], RECOPE TRANSPORTES, FINEP, 59-86. 
(47) Maculan, N.; Passini, M.M.; Brito, J.A.M. \& Lisser, A. (2002). Column generation methods for network design. In: Transportation and Network Analysis: Current Trends [edited by M. Gendreau and P. Marcotte], 165-179, Kluwer.

(48) Maculan, N. \& Ferraz Pereira, M.V. (1980). Programação linear. Editora Atlas S.A.

(49) Maculan, N.; Plateau, G. \& Lisser, A. (2003). Integer linear models with a polynomial number of variables and constraints for some classical combinatorial optimization problems. Pesquisa Operacional, 23(1), 161-168.

(50) Maculan, N.; Porto, S.C.S.; Ribeiro, C.C. \& Souza, C.C. de (1999). A new formulation for scheduling unrelated processors under precedence constraints. RAIRO Rceherche Opérationelle, 33, 87-92.

(51) Maculan, N.; Prata Santiago, C.; Macambira, E.M. \& Jardim, M.H.C. (2002). An O(n) algorithm for projecting a vector on the intersection of a hyperplane and a box in $\mathrm{R}^{\mathrm{n}}$. Journal of Optimization Theory and Applications, a ser publicado.

(52) Maculan, N. \& Salles, J.J.C. (1991). A lower bound for the shortest Hamiltonean path in directed graphs. OR Spektrum, 13, 99-102.

(53) Maculan, N.; Souza, M. \& Candia-Vejar, A. (1991). An approach for the Steiner problem in directed graphs. Annals of Operations Research, 33, 471-480.

(54) Maculan, N.; Souza, P. \& Candia Vejar, A. (1993). Reduction tests for the Steiner problem in directed graphs. Pesquisa Operacional, 11(2), 48-56.

(55) Maculan, N. \& Villares, M.L. (1981). Comentários sobre um algoritmo para a Solução Aproximada do Problema da Mochila. Pesquisa Operacional, 1(1), 23-28.

(56) Mauricio, D. \& Maculan, N. (1994). O método feixe nível proximal para problemas min-max e otimização convexa não suave. Trabajos Seleccionados del VII CLAIO Congreso Latino-Ibero-Americano de Investigación Operativa e Ingeniería de Sistemas, Santiago de Chile, 419-437.

(57) Mauricio, D. \& Maculan, N. (1997). A relationship between global optimization and 0-1 nonlinear programming. Pesquisa Operacional, 17(2), 165-172.

(58) Mauricio, D. \& Maculan, N. (1997). A trust region method for zero-one nonlinear programming. RAIRO Recherche Opérationnelle, 31, 331-341.

(59) Mauricio, D. \& Maculan, N. (2000). A Boolean penalty method for zero-one nonlinear programming. Journal of Global Optimization, 16, 343-354.

(60) Michelon, P. \& Maculan, N. (1991). Lagrangean decomposition for integer nonlinear programming with linear constraints. Mathematical Programming, 52, 303-313.

(61) Michelon, P. \& Maculan, N. (1993). Lagrangean methods for 0-1 quadratic problems. Discrete Applied Mathematics, 42, 257-269.

(62) Michelon, P.; Ripeau, S. \& Maculan, N. (2001). Un algorithme pour la bipartition d' un graphe en sous-graphes de cardinalité fixée. RAIRO Recherche Opérationnelle, 35, 401-414.

(63) Maculan, N.; Michelon, P. \& Plateau, G. (1992). Column-generation in linear programming with bounding variable constraints and its application in integer programming. Pesquisa Operacional, 12(2), 45-57. 
(64) Mondaini, R.; Freire-Mondaini, D. \& Maculan, N. (1998). The study of Steiner points associated with the vertices of regular tetrahedra joined together at common faces. Investigación Operativa, 6, 103-110.

(65) Montenegro, F.; Maculan, N.; Plateau, G. \& Boucher, P. (2001). New heuristics for the Euclidean Steiner problem in $\mathrm{R}^{\mathrm{n}}$. In: Essays and Surveys in Metaheuristics [edited by C.C. Ribeiro and P. Hansen], 509-524, Kluwer.

(66) Nagih, A.; Plateau, A.; Plateau, G. \& Maculan, N. (2002). Programmation linéaire en nombres entiers. In: Outils d'analyse numérique pour l'automatique [edited by A. Barraud], Capítulo 8, 247-344, Hermès.

(67) Negreiros Gomes, M.J.; Maculan, N.; Negreiros Gomes, F.J. \& Ochi, L.S. (1994). Resolvendo problemas de distribuição de derivados de petróleo com SisGRAFOS. Trabajos Seleccionados del VII CLAIO - Congreso Latino-Ibero-Americano de Investigación Operativa e Ingeniería de Sistemas, Santiago de Chile, 297-305.

(68) Negreiros Gomes, M.J.; Maculan, N. \& Ochi, L.S. (1996). O problema de compras. Pesquisa Operacional, 14(2), 73-86.

(69) Ochi, L.S.; Santos, E.M.; Montenegro, A.A. \& Maculan, N. (1995). Genetic algorithm for the travelling purchaser problem. In: Proceedings of the Metaheurictics International Conference [edited by I.H. Osman and J.P. Kelly], 52-57, Kluwer.

(70) Ochi, L.S.; Maculan, N. \& Figueiredo, R.M.V. (1998). A new self-organizing strategy based on elastic networks for solving the Euclidean traveling salesman problem. Lectures Notes in Artificial Intelligence, 1416, 479-487.

(71) Ortiz, C.; Maculan, N. \& Szwarcfiter, J.L. (1998). Characterizing and edge-coloring split-difference graphs. Discrete Applied Mathematics, 82, 209-217.

(72) Parada-Daza, V. \& Maculan, N. (1995). Un nuevo método de solución para el problema de equilibrio de tráfico generalizado. Revista del Instituto Chileno de Investigación de Operaciones, 4(2), 29-41.

(73) Paula Jr., G.G. de \& Maculan, N. (1978). Uma experiência com a utilização de filtros em programação inteira bivalente. Anais do XI Simpósio Brasileiro de Pesquisa Operacional.

(74) Paula Jr., G.G. de \& Maculan, N. (1978). Desenvolvimento de uma metodologia para a solução de alocação ótima de tripulação em rotas aéreas. Anais do XI Simpósio Brasileiro de Pesquisa Operacional.

(75) Paula Jr., G.G. de \& Maculan, N. (1988). Análise do aspecto combinatório da localização dinâmica de carvoarias em plantações de eucaliptos. Revista Ceres, 35, 388-399.

(76) Paula Jr., G.G. de \& Maculan, N. (1989). Uma heurística de decomposição primal para a solução de problemas não capacitados de localização de p-medianas. Experientiae, 30(10), 123-134.

(77) Pradenas-Rojas, L. \& Maculan, N. (1993). Un método de solución para el problema de camino mínimo con múltiples objetivos. Investigación Operativa, 3, 191-200.

(78) Reinoso, H. \& Maculan, N. (1992). Lagrangean decomposition in integer linear programming: A new scheme. INFOR, 30, 1-5. 
(79) Ribeiro, C.C. \& Maculan, N. (editores, 1994). Applications of Combinatorial Optimization. (Annals of Operations Research, 50). J.C. Baltzer.

(80) Rocha, T.G.; Almeida, R.; Moreno, A. \& Maculan, N. (1981). The administration of standard length telephone cable reels. Annals of Discrete Mathematics, 11, 109-123.

(81) Rocha, T.G.; Almeida, R.; Moreno, A. \& Maculan, N. (1981). On the solution of the standard length telephone cable reels problem. In: Operational Research'81 [edited by J.P. Brans], 825-839, North Holland.

(82) Rozenthal, M.; Carvalho, L.A.V.; Laks, J.; Maculan, N. \& Engelhardt, E. (2000). Searching and analysing neuropsychological patterns in schizophrenia with artificial neural networks. Jornal Brasileiro de Psiquiatria, 49(1-2), 5-12.

(83) Rozenthal, M.; Lages, D.D.; Engelhardt, E. \& Maculan, N. (2000). Desenvolvimento de aplicativo usando inteligência artificial para classificação de pacientes esquizofrênicos segundo perfis neuropsicológicos. Arquivos Brasileiros de Psiquiatria, Neurologia e Medicina Legal, 74, 39-42.

(84) Souza, M.J.F.; Maculan, N. \& Ochi, L.S. (2000). Melhorando quadros de horário de escolas através de caminhos mínimos. Tendências em Matemática Aplicada e Computacional, 1, 515-524.

(85) Souza, M.J.F.; Maculan, N. \& Ochi, L.S. (2001). Uma heurística para o problema de programação de horários em escolas. Tendências em Matemática Aplicada e Computacional, 2, 213-222.

(86) Thomaz, A.C.F.; Gomes, F.J. \& Maculan, N. (1984). Controle ótimo em planejamento de capturas de lagostas no Nordeste do Brasil. Anais do XVIII Simpósio Brasileiro de Pesquisa Operacional, 354-361.

(87) Thomaz, A.C.F.; Gomes, F.J.; Craveiro de Souza, R.; Oliveira, A.A. \& Maculan, N. (1986). Optimal scenario for waste collection in Fortaleza city: An application of the chinese-postman problem. Anais do XIX Simpósio Brasileiro de Pesquisa Operacional, 109-117.

(88) Vaca-Obando, F.E.; Feigenbaum, D.; Cosenza, C.A.N. \& Maculan, N. (1975). Metodologia geral para abordar um problema de políticas alternativas de transporte numa região. Anais do VIII Simpósio Brasileiro de Pesquisa Operacional.

(89) Vaca-Obando, F.E.; Villares, M.L. \& Maculan, N. (1978). Tópicos de dualidade em programação inteira bivalente. Anais do II Congresso Brasileiro de Automática, 495-509.

(90) Vaca-Obando, F.E.; Villares, M.L. \& Maculan, N. (1980). Soluções aproximadas do problema de programação bivalente utilizando dualidade em programação inteira. Revista de Informática e Investigación Operativa, 40, 53-85.

(91) Villares, M.L. \& Maculan, N. (1982). Variáveis ordenadas por grupos. Pesquisa Operacional, 2(2), 29-48.

(92) Xavier, A.E. \& Maculan, N. (1986). Extrapolação em penalização hiperbólica. Avances en Investigación Operativa, 24-38, ADIO, Buenos Aires.

(93) Yanasse, H.H.; Soma, N.Y. \& Maculan, N. (2000). An algorithm for determining the k-best solutions of the one-dimensional knapsack problem. Pesquisa Operacional, 20(1), 117-134. 


\section{Anexo II: Depoimento de ex-alunos}

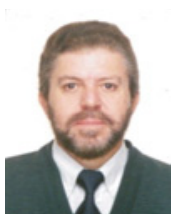

"Sobre ele afirmo que a história registrará o quanto este país lhe deve pela imensa contribuição ao progresso da Otimização Combinatória que nasceu de seu esforço e talento. Sua estatura moral e científica continua a procura de um par, de um termo de comparação. Respeito da comunidade científica internacional? Desse, sou testemunha, nas vezes em que com ele viajei pelo mundo." (Geraldo Galdino de Paula Junior, UENF, Campos)

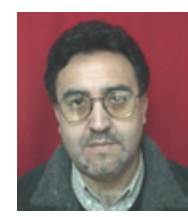

"Eu gostaria de destacar algumas facetas de Nelson: a primeira é a sua capacidade de liderança, ele é quem eu posso chamar de um líder natural. Também devo destacar sua constante preocupação com seus alunos, tanto no nível acadêmico quanto no pessoal e humano. Não foram poucas as vezes que ele nos deu algum suporte especial. Particularmente, devo agradecer ao Nelson de ter me iniciado em pesquisa de alto nível, além de me deixar uma marca importante de vida como fonte de estímulo e inspiração." (Alfredo Enrique Candia-Vejar, Universidad de Talca, Chile)

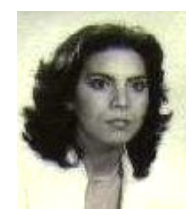

"Maculan foi importante e vital na minha vida. Conheci-o na PUC do Rio de Janeiro ainda como estudante de mestrado. Logo tive a felicidade de têlo como meu orientador de doutorado. Naquela ocasião, mesmo como reitor da UFRJ, nunca deixou de acompanhar meus trabalhos, atendendome em sua própria casa, mesmo após um longo dia de difíceis tarefas administrativas. Lembro-me do Nelson como excelente professor de Programação Inteira, recebendo na sala de aula estudantes que chegavam direto do aeroporto com as malas nas mãos. Maculan socorria a todos, providenciando-lhes acomodação, quando não avalizava para eles o aluguel da primeira moradia carioca, permitindo-lhes estudar com tranqüilidade. Com o seu exemplo e orientação nos iniciou não só na pesquisa científica, como nos permitiu atuar na administração universitária. Todos os orientados chilenos do Nelson têm sido chefes de departamentos e alguns deles vêm sendo gestores de cursos de pósgraduação em diferentes universidades do Chile." (Lorena Del Carmen Pradena Rojas, Universidad de Concepción, Chile)

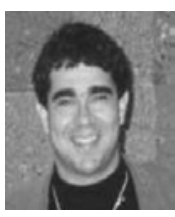

"Fui premiado pela amizade do professor ao longo de sua orientação e até os dias de hoje. Neste tempo, tive o privilégio de conhecer uma pessoa ímpar neste País. Maculan é um sério lutador pelas causas da academia e um grande colaborador da melhoria científica do País. É um exemplo de vida que busco passar adiante." (Marcos José Negreiro Gomes, UECE/CEFET, Fortaleza) 


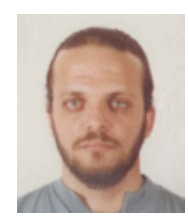

"No mundo da pesquisa, fortes pressões nos impelem a dois caminhos fáceis: vaidade acadêmica, que paralisa o potencial criativo e de crescimento, e o comércio de idéias, que destrói todas as aspirações e longo prazo. Nos anos que convivi como aluno com o Professor Maculan recebi orientação científica, segura e inspiradora, mas aprendi também a reconhecer e evitar os caminhos fáceis; aprendi a olhar o mundo e a enxergar nele meu lugar de pesquisador, cidadão e ser humano." (Marcos Passini, UFRJ, Rio de Janeiro)

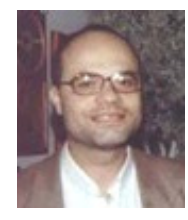

"A confiança que adquiri junto ao Nelson, à sua pessoa marcante e inconfundível, é hoje, para mim, uma conquista ainda maior que o conhecimento que pude obter ao seu lado. A meu amigo Maculan expresso meu sincero respeito, admiração e carinho." (Robinson Samuel Vieira Hoto, UEL, Londrina)

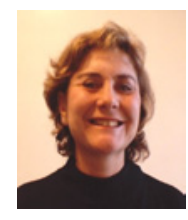

"Mestre Maculan ensinou-me Modelagem e Programação Inteira de Grande Porte. Ensinou-me que a Matemática, tão abstrata, é fruto concreto da História. Ensinou-me que a função do Professor é de transmitir o conhecimento de maneira que possa ser aprendido e de ensinar a pensar de maneira crítica. Paranaense, ensinou-me que o Brasil é composto de mais do que dois estados e que a América Latina é formada de mais do que três países. Intelectual, ensinou-me que um dos principais papéis do intelectual é de criticar e combater a lógica fria do poder, respeitando ao mesmo tempo as nossas instituições legais, tão duramente conquistadas. Humanista, ensinou-me a importância de Leibnitz, Pascal, Voltaire, da Vinci, Flaubert, Cecília Meirelles, Clarice Lispector, Guimarães Rosa, Octavio Paz, Garcia Marquez, Portinari, Villa-Lobos, Fernanda Montenegro e de tantos outros. Ensinou-me que podemos ser sofisticados dentro da simplicidade, rigorosos e gentis; que o sorriso não destrói a seriedade; que se pode torcer pelo América num mundo flamenguista; que podemos criar, fazer projetos, reiniciar aos 60 anos. Abriu as portas para as mulheres em instituições machistas por excelência. Ensinou-me que os velhos são os nossos melhores professores e que o mundo depende dos jovens. Mestre Maculan, expressão tantas vezes com carinho repetida, deveria ser considerada um pleonasmo." (Dina Feigenbaum, Technotrade, Canadá)

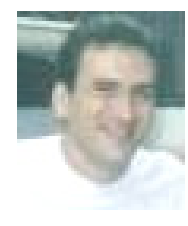

"O Professor Maculan é uma figura ímpar que tem dado uma contribuição extraordinária à formação de recursos humanos em Pesquisa Operacional, sobretudo na área de Otimização Combinatória. Além de um grande pesquisador é ser humano admirável que tem se caracterizado pela maneira sempre gentil, educada e prestativa com que orienta seus alunos." (Lucídio dos Anjos Formiga Cabral, UFPa, João Pessoa) 


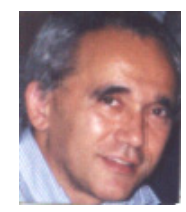

"Muito pode ser dito a propósito de Maculan, vou me concentrar em uma de suas qualidades, que considero vital para a comunidade acadêmica liderança científica. Ou seja, a capacidade de reunir pessoas em torno de um tema interessante e promissor, provendo os meios intelectuais e materiais para que ele seja desenvolvido." (Luis Paulo Vieira Braga, UFRJ, Rio de Janeiro)

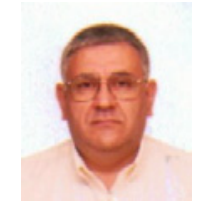

"O intercâmbio técnico e científico com todos os seus orientados sempre foi evidente. No entanto, gostaria de destacar o lado humano do grande cientista. Eu me lembro da forma como ele costumava acolher os seus alunos e orientados, de como fazia o possível para ajudá-los, em todos os sentidos, não somente no aspecto acadêmico, o que, em mais de uma ocasião, deve ter-lhe custado alguns dissabores." (Oscar Ivan Palma Pacheco, Embrapa, Rio de Janeiro)

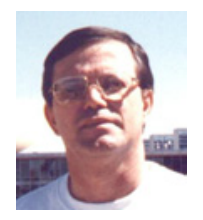

"Ao mestre, com carinho, os meus mais sinceros e profundos agradecimentos. Pela orientação tão dedicada que sempre deu ao meu trabalho. Pelo exemplo de paciência e perseverança, que me fizeram continuamente tentar alcançar o meu objetivo, encontrar a minha solução ótima. Agradeço, também, pela otimização de horários, escalonamento de compromissos, enfim, todos os esforços que realizou com a intenção de sempre estar presente e pronto a ajudar-me quando eu encontrava dificuldades. Este espírito prestativo e amigo estará armazenado na minha mente para sempre. Parabéns!" (Dario José Aloise, UFRN, Natal)

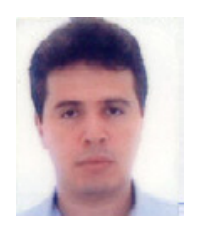

"O Professor Maculan é uma das pessoas mais inteligentes e dedicadas que conheço. Tenho por ele grande apreço e admiração, não só por sua excelência acadêmica e científica, mas, também, pela humanidade e grandeza de atitudes com que exerce sua liderança. Agradeço-o por seus ensinamentos, seu exemplo de conduta profissional e toda a inestimável ajuda que sempre prestou a nós, seus alunos e colegas, na superação dos diversos obstáculos da difícil atividade de pesquisa." (Flávio Marcelo Tavares Montenegro, UFRJ, Rio de Janeiro)

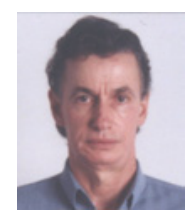

"Entendo que é desnecessário destacar a importância do Professor Nelson, como elemento de referência para nossa área de otimização, dada a sua extensa contribuição acadêmica e científica para o desenvolvimento da mesma. Parabéns Nelson e mais sucesso nesta sua caminhada." (Arlindo Gomes de Alvarenga, UFES, Vitória) 


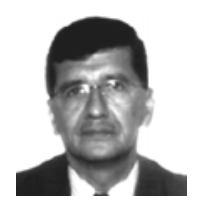

"Em todas as atividades de sua vida, seus colegas e alunos consideravam Maculan como um amigo incondicional e um excelente solucionador de problemas. Tanto dos problemas científicos como de problemas humanos, muitas vezes à custa de sacrifícios pessoais. Quantas vezes ele forneceu ajuda financeira a seus estudantes, não só aos brasileiros, mas sobretudo aos estrangeiros, que muitas vezes precisavam do seu aval para as primeiras instalações no Rio de Janeiro. Gostaria de resumir a personalidade deste grande brasileiro nas seguintes palavras em casteliano: Nelson Maculan es un maestro investigador a tiempo completo y un ser humano expectacular." (Félix Eduardo Vaca Obando, Escuela Politecnica Nacional, Equador)

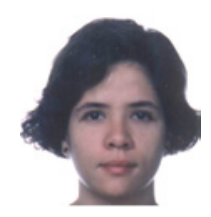

"Trabalhar com o Professor Nelson Maculan é extremamente gratificante, não apenas por seu vasto conhecimento, mas principalmente por sua criatividade e pelo interesse com que ele acompanha o trabalho de pesquisa de cada orientando. Ademais, não posso deixar de ressaltar sua dedicação aos alunos e ao ensino de um modo geral. Entretanto, uma característica faz de Nelson Maculan uma pessoa diferente de todas as demais: seu grande coração. Meu grande amigo, conselheiro, uma pessoa realmente admirável com quem tenho a honra de trabalhar e conviver." (Laura Sílvia Bahiense da Silva Leite, PSR, Rio de Janeiro)

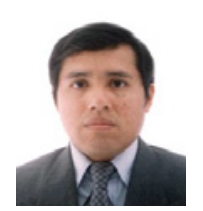

"O professor e amigo Nelson Maculan, em minha opinião, é um dos pioneiros da Pesquisa Operacional no Brasil e um dos principais formadores desta área na América Latina. Sua excelência acadêmica, representada por importantes e diversas publicações, é o mais importante legado para a formação de recursos humanos comprometidos com a área e com o desenvolvimento nacional, ultrapassando as fronteiras brasileiras, se estendendo de forma representativa na América Latina." (David Santos Mauricio Sanchez, Universidad Nacional Mayor de San Marcos, Perú)

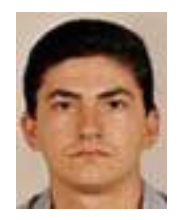

"Seu apoio e incentivo foram fundamentais para a minha formação científica. Destaco o seu exemplo de dedicação e sua coragem para enfrentar problemas novos e desafiadores." (Carlile Campos Lavor, UFF, Niterói)

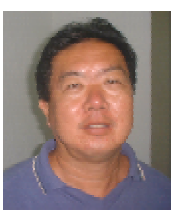

"Durante o doutorado tive o privilégio de contar com um orientador com idéias fantásticas sobre diferentes assuntos da área de computação e matemática aplicada. Ele não foi somente um orientador de doutorado, mas também um exemplo de mestre e grande amigo." (Luiz Satoru Ochi, UFF, Niterói) 


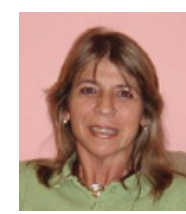

"A sorte de conviver com Maculan, deixa-me em dívida com a vida. Sua conduta e sua simplicidade, mesmo com toda inteligência privilegiada e o reconhecido destaque científico, é mais que exemplo, é lição de vida. Esse é um amigo de todos os segundos, de solidariedade incondicional e generosidade sistemática." (Maria Helena Cautiero Horta Jardim, UFRJ, Rio de Janeiro)

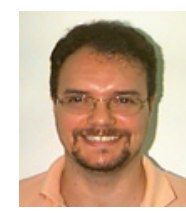

"Além da qualidade como pesquisador e da dedicação exemplar do Prof. Maculan, deve-se ressaltar também sua conduta e postura como pessoa humana. Certamente, mais do que ele próprio perceba, nos ensinou a atuar como verdadeiros profissionais, indo muito além das questões puramente técnicas." (Carlos Alberto Martinhon, UFF, Niterói)

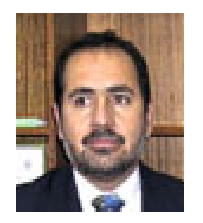

"Maculan vem conduzindo a América Latina no caminho do progresso em Otimização. Estarei sempre agradecido a ele por isso e também pela oportunidade que me deu de navegar no mundo do conhecimento." (Victor Manuel Parada Daza, USACH, Chile)

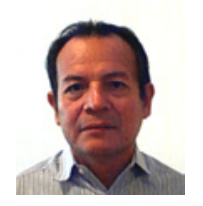

"Escrever sobre o Professor Maculan deveria ser fácil levando-se em conta a sua excelente qualidade humana e o seu vasto trabalho, amplamente reconhecido pela comunidade acadêmica. Porém, resulta difícil tentar expressar em poucas palavras o meu agradecimento ao mestre, orientador e amigo." (Luis Ernesto Torres Guardia, UFF, Niterói)

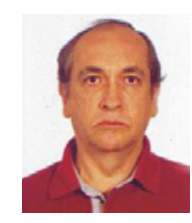

"Poucos certamente serão os professores em todo o Brasil que tenham orientado tantos mestres e tantos doutores, em um espectro tão amplo de conhecimentos, contemplando temas ligados às mais diversas especializações da Programação Matemática, da Engenharia, da Computação, da Estatística, da Economia ou da Educação. Na minha opinião o mais destacável é que essa orientação, em quase sua totalidade, não se cinge aos estritos campos da pesquisa temática central, mas extravasa às mais diversas questões culturais, científicas e existenciais, onde a dimensão humanista e enciclopedista do Professor Maculan se avulta e contrasta de forma diferenciada." (Adilson Elias Xavier, UFRJ, Rio de Janeiro) 


\section{Anexo III: Árvore dos Doutores Orientados}

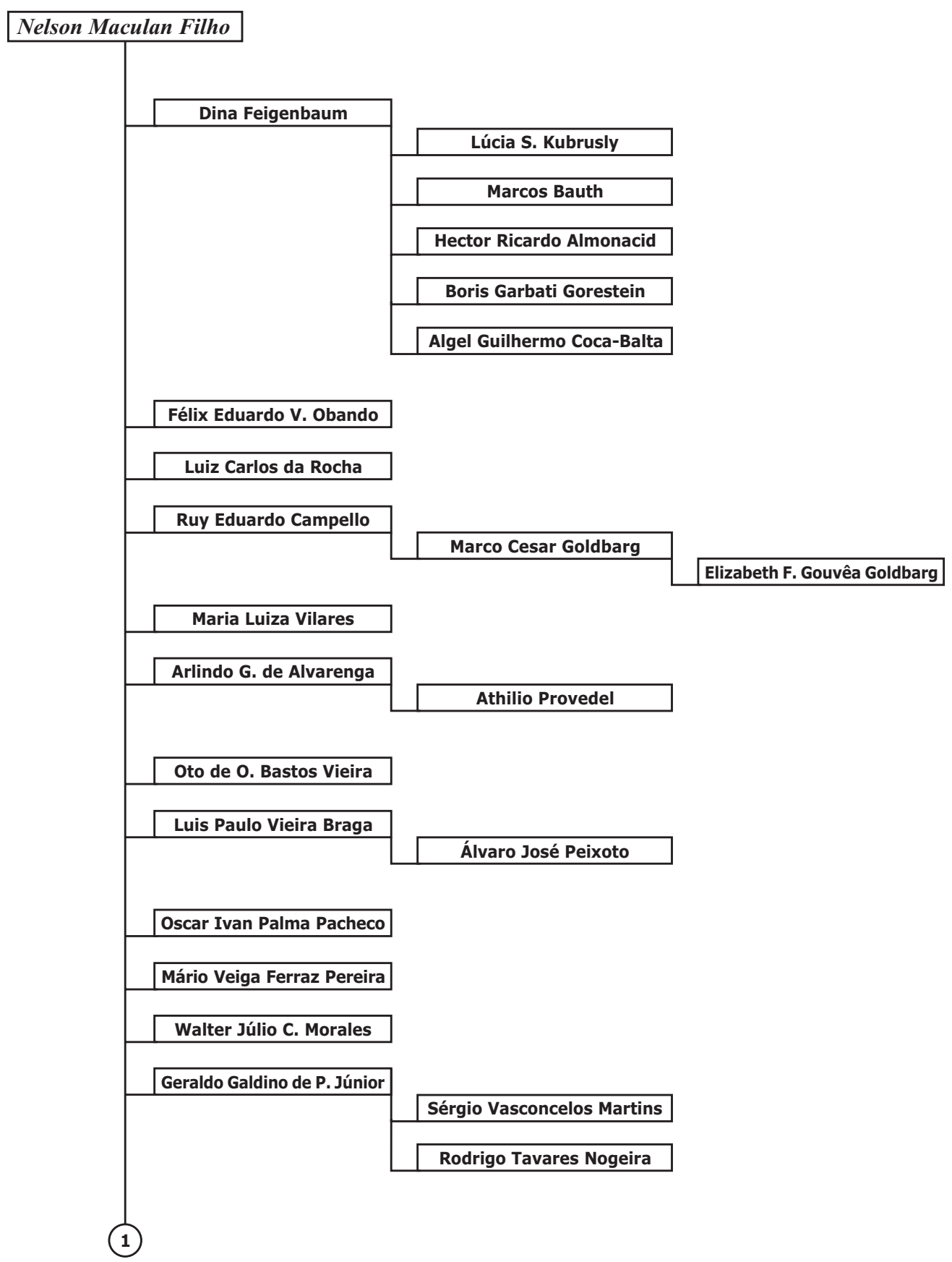




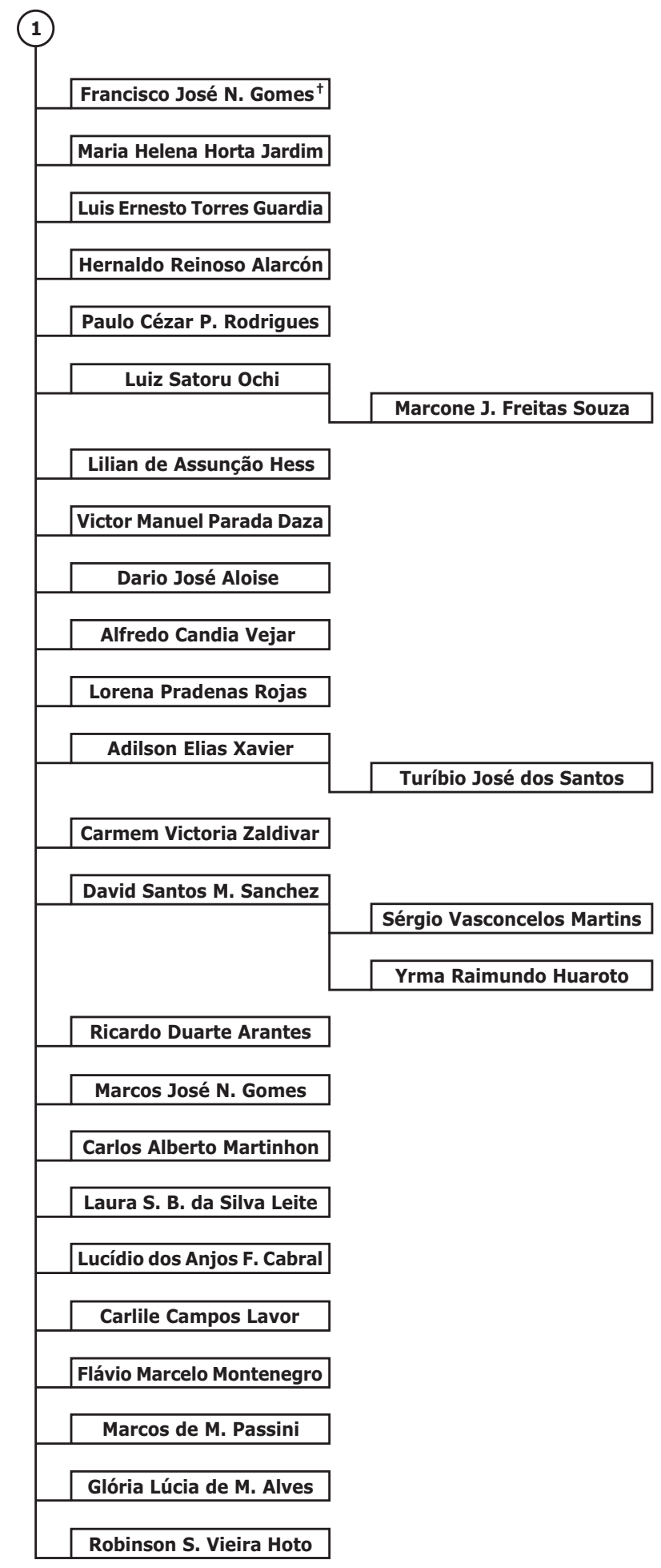

